PROCEEDINGS OF THE

AMERICAN MATHEMATICAL SOCIETY

Volume 126, Number 8, August 1998, Pages 2369-2376

S 0002-9939(98)04323-8

\title{
FIRST ORDER DIFFERENTIAL OPERATORS IN WHITE NOISE ANALYSIS
}

\author{
DONG MYUNG CHUNG AND TAE SU CHUNG
}

(Communicated by Palle E. T. Jorgensen)

\begin{abstract}
Let $(E)$ be the space of test white noise functionals. We first introduce a family $\left\{\diamond_{\gamma} ; \gamma \in \mathbb{C}\right\}$ of products on $(E)$ including Wiener and Wick products, and then show that with each product $\diamond_{\gamma}$, we can associate a first order differential operator, called a first order $\gamma$-differential operator. We next show that a first order $\gamma$-differential operator is indeed a continuous derivation under the product $\diamond_{\gamma}$. We finally characterize $\gamma \Delta_{G}+N$ by means of rotation-invariance and continuous derivation under the product $\diamond_{\gamma}$. Here $\Delta_{G}$ and $N$ are the Gross Laplacian and the number operator on $(E)$, respectively.
\end{abstract}

\section{INTRODUCTION}

The white noise analysis, initiated by Hida in 1975, has been developed into an infinite dimensional distribution theory with applications to quantum physics, infinite dimensional harmonic analysis, stochastic analysis and so on. The mathematical framework of white noise analysis is the Gel'fand triple $(E) \subset\left(L^{2}\right) \subset(E)^{*}$ over Gaussian space $\left(E^{*}, \mu\right)$, where $\mu$ is the standard Gaussian measure associated with a Gel'fand triple $E \subset H \subset E^{*}$.

Infinite dimensional Laplacians based on white noise analysis, in particular the Gross Laplacian $\Delta_{G}$ and the number operator $N$, have been considerably studied, see e.g. [5], [7], [8], [9]. In [8], Obata showed that $\Delta_{G}$ and $N$ are rotation-invariant. In [5], Hida, Kuo and Obata proved that $\frac{i}{2} \Delta_{G}+i N$ is the infinitesimal generator of the one-parameter group of adjoint Kuo's Fourier-Mehler transforms. In [3], Chung and Ji showed that for each $a, b \in \mathbb{C}$, we can obtain explicitly a one-parameter transformation group with infinitesimal generator $a \Delta_{G}+b N$. A main purpose of this paper is to study characteristic properties of operators of the form $\gamma \Delta_{G}+N, \gamma \in \mathbb{C}$.

It is well-known (see [7]) that the Wiener product and the Wick product have the derivation property on $(E)$. In [10], Obata introduced the concept of first order differential operators with variable coefficients and then showed that a first order differential operator with variable coefficients is indeed a continuous derivation under the Wiener product. In [1], Chung and Chung introduced the concept of first order Wick differential operators with variable coefficients and then showed that a first order Wick differential operator with variable coefficients is indeed a continuous derivation under the Wick product.

Received by the editors January 21, 1997.

1991 Mathematics Subject Classification. Primary 46F25.

Research supported by KOSEF 996-0100-00102 and BSRI 97-1412.

(c)1998 American Mathematical Society 
In this paper, motivated by [1] and [10], we first introduce a family $\left\{\diamond_{\gamma} ; \gamma \in \mathbb{C}\right\}$ of products on $(E)$ including Wiener and Wick products, and then show that with each product $\diamond_{\gamma}$, we can associate a first order differential operator, called a first order $\gamma$-differential operator. We next show that a first order $\gamma$-differential operator is indeed a continuous derivation under the product $\diamond_{\gamma}$. We finally characterize $\gamma \Delta_{G}+N$ by means of rotation-invariance and continuous derivation under the product $\diamond_{\gamma}$.

\section{Preliminaries}

Let $T$ be a topological space with a Borel measure $d \nu(t) \equiv d t$, and assume that $H \equiv L^{2}(T, \nu ; \mathbb{R})$ is a real separable Hilbert space with norm $|\cdot|_{0}$. Let $A$ be a positive self-adjoint operator on $H$ such that $\rho \equiv\left\|A^{-1}\right\|_{\mathrm{OP}}<1$ and $\left\|A^{-1}\right\|_{\mathrm{HS}}<\infty$. With this $A$, a Gel'fand triple $E \subset H \subset E^{*}$ is constructed in the standard manner (see [9]). Recall that $E$ is a countable Hilbert nuclear space induced by a family of Hilbert norms: $|\xi|_{p}=\left|A^{p} \xi\right|_{0}, \xi \in H, p \in \mathbb{R}$.

Let $\mu$ be the standard Gaussian measure on $E^{*}$, i.e., its characteristic function is given by $e^{-\frac{1}{2}|\xi|_{0}^{2}}, \xi \in E$. Let $\left(L^{2}\right)$ be the space of $\mathbb{C}$-valued $\mu$-square integrable functions on $E^{*}$. Then by the Wiener-Itô decomposition theorem, each $\phi \in\left(L^{2}\right)$ admits a unique expansion

$$
\phi(x)=\sum_{n=0}^{\infty}\left\langle: x^{\otimes n}:, f_{n}\right\rangle, \quad x \in E^{*}, f_{n} \in H_{\mathbb{C}}^{\widehat{\otimes} n},
$$

where : $x^{\otimes n}$ : is a Wick ordering of $x^{\otimes n}$ (see [6], [7]) and $H_{\mathbb{C}}^{\widehat{\otimes} n}$ is the $n$-fold symmetric tensor product of the complexification of $H$. In this case, we simply write $\phi \sim\left(f_{n}\right)$.

Let $(E)$ be the space of $\phi \sim\left(f_{n}\right) \in\left(L^{2}\right)$ such that $f_{n} \in E_{\mathbb{C}}^{\widehat{\otimes} n}$ for all $n$, and $\|\phi\|_{p}^{2} \equiv \sum_{n=0}^{\infty} n !\left|f_{n}\right|_{p}^{2}<\infty$ for all $p \geq 0$. Then we have a Gel'fand triple: $(E) \subset$ $\left(L^{2}\right) \subset(E)^{*}$, where $(E)^{*}$ is the strong dual space of $(E)$. Moreover, it is known that for each $\Phi \in(E)^{*}$ there exists a unique sequence $\left\{F_{n}\right\}_{n=0}^{\infty}$ with $F_{n} \in\left(E_{\mathbb{C}}^{\otimes n}\right)_{\text {sym }}^{*}$ such that

$$
\langle\langle\Phi, \phi\rangle\rangle=\sum_{n=0}^{\infty} n !\left\langle F_{n}, f_{n}\right\rangle, \quad \phi \sim\left(f_{n}\right) \in(E),
$$

and $\|\Phi\|_{-p}^{2} \equiv \sum_{n=0}^{\infty} n !\left|F_{n}\right|_{-p}^{2}<\infty$ for some $p \geq 0$. In this case we also write $\Phi \sim\left(F_{n}\right)$. These elements $\phi \in(E)$ and $\Phi \in(E)^{*}$ are called a test (white noise) functional and a generalized (white noise) functional, respectively.

For $\xi \in E_{\mathbb{C}}$ an exponential vector $\varphi_{\xi}$ is defined by $\varphi_{\xi} \sim\left(\frac{1}{n !} \xi^{\otimes n}\right)$. The $S$-transform of $\Phi \in(E)^{*}$ is a function on $E_{\mathbb{C}}$ defined by

$$
S \Phi(\xi)=\left\langle\left\langle\Phi, \varphi_{\xi}\right\rangle\right\rangle, \quad \xi \in E_{\mathbb{C}} .
$$

Let $\mathcal{L}\left((E),(E)^{*}\right)$ (resp. $\left.\mathcal{L}((E),(E))\right)$ be the space of all continuous linear operators from $(E)$ into $(E)^{*}$ (resp. $(E)$ ). For $\Xi \in \mathcal{L}\left((E),(E)^{*}\right)$ the symbol of $\Xi$ is a function on $E_{\mathbb{C}} \times E_{\mathbb{C}}$ defined by

$$
\widehat{\Xi}(\xi, \eta)=\left\langle\left\langle\Xi \varphi_{\xi}, \varphi_{\eta}\right\rangle\right\rangle, \quad \xi, \eta \in E_{\mathbb{C}} .
$$

Theorem 2.1 ([2], [9]). Suppose that a $\mathbb{C}$-valued function $F$ on $E_{\mathbb{C}} \times E_{\mathbb{C}}$ satisfies the following conditions:

(S1) For any $\xi, \xi^{\prime}, \eta$ and $\eta^{\prime}$ in $E_{\mathbb{C}}$, the function $(z, w) \mapsto F\left(z \xi+\xi^{\prime}, w \eta+\eta^{\prime}\right)$ is an entire function on $\mathbb{C} \times \mathbb{C}$. 
(S2) There exist $p \geq 0, a>0$ and $K>0$ such that

$$
|F(\xi, \eta)| \leq K e^{a\left(|\xi|_{p}^{2}+|\eta|_{p}^{2}\right)}, \quad \xi, \eta \in E_{\mathbb{C}} .
$$

Then there exists a unique $\Xi \in \mathcal{L}\left((E),(E)^{*}\right)$ such that $F$ is the symbol of $\Xi$. Moreover, for $q>p$ with $2 a e^{2}\left\|A^{-2(q-p)}\right\|_{\mathrm{HS}}^{2}<1$, we have

$$
\|\Xi \phi\|_{-q} \leq K\left(1-2 a e^{2}\left\|A^{-2(q-p)}\right\|_{\mathrm{HS}}^{2}\right)^{-1}\|\phi\|_{q}, \quad \phi \in(E) .
$$

It is known that there exist continuous linear operators $\Delta_{G}, N \in \mathcal{L}((E),(E))$ such that $\widehat{\Delta_{G}}(\xi, \eta)=e^{\langle\xi, \eta\rangle}\langle\xi, \xi\rangle$ and $\widehat{N}(\xi, \eta)=e^{\langle\xi, \eta\rangle}\langle\xi, \eta\rangle, \xi, \eta \in E_{\mathbb{C}}$. These are called the Gross Laplacian and the number operator, respectively.

For $\alpha, \beta \in \mathbb{C}$, the $\mathcal{G}_{\alpha, \beta}$-transform acting on $(E)$ is defined by

$$
\mathcal{G}_{\alpha, \beta} \varphi_{\xi}=e^{\alpha\langle\xi, \eta\rangle} \varphi_{\beta \xi}, \quad \xi \in E_{\mathbb{C}} .
$$

It is known [3] that $\mathcal{G}_{\alpha, \beta} \in \mathcal{L}((E),(E))$ and $\left\{\mathcal{G}_{\alpha, \beta} ; \alpha, \beta \in \mathbb{C}, \beta \neq 0\right\}$ forms a twoparameter transformation group with generators $\Delta_{G}$ and $N$. Moreover, we note that $\mathcal{G}_{\alpha^{\prime}, \beta^{\prime}} \mathcal{G}_{\alpha, \beta}=\mathcal{G}_{\alpha+\alpha^{\prime} \beta^{2}, \beta \beta^{\prime}}, \mathcal{G}_{0,1}=I$ and $\mathcal{G}_{\alpha, \beta}^{-1}=\mathcal{G}_{-\alpha \beta^{-2}, \beta^{-1}}, \beta \neq 0$.

\section{3. $\gamma$-PRODUCT ON WHITE NOISE FUNCTIONALS}

It is known [7], [9] that the Wiener product (pointwise multiplication) is a continuous binary operation on $(E)$.

For $\Phi, \Psi \in(E)^{*}$, the Wick product $\Phi \diamond \Psi \in(E)^{*}$ is defined by $S(\Phi \diamond \Psi)=S \Phi \cdot S \Psi$. It is well-defined, thanks to the characterization theorem for $(E)^{*}$ given in [11]. Note that the Wick product is also a continuous binary operation on $(E)$.

Throughout this paper, let $\gamma$ be a fixed but arbitrary complex number.

Proposition 3.1. There exists a unique continuous binary operation $\diamond_{\gamma}$ on $(E)$ such that

$$
\varphi_{\xi} \diamond_{\gamma} \varphi_{\eta}=e^{\gamma\langle\xi, \eta\rangle} \varphi_{\xi+\eta}, \quad \xi, \eta \in E_{\mathbb{C}} .
$$

Proof. Let $\alpha, \beta \in \mathbb{C}$ with $\gamma=\beta^{2}-2 \alpha, \beta \neq 0$. Define a binary operation $\diamond_{\gamma}$ on $(E)$ by

$$
\phi \diamond_{\gamma} \psi=\mathcal{G}_{\alpha, \beta}^{-1}\left(\mathcal{G}_{\alpha, \beta} \phi \cdot \mathcal{G}_{\alpha, \beta} \psi\right), \quad \phi, \psi \in(E) .
$$

Then by the continuity of $\mathcal{G}_{\alpha, \beta}$ and the continuity of the Wiener product, we see that $\diamond_{\gamma}$ is continuous. We easily see that (3.1) is verified by a direct computation. Moreover, since $\left\{\varphi_{\xi} ; \xi \in E_{\mathbb{C}}\right\}$ spans a dense linear subspace of $(E), \diamond_{\gamma}$ is welldefined for any choice of $\alpha, \beta \in \mathbb{C}$ satisfying $\gamma=\beta^{2}-2 \alpha, \beta \neq 0$.

We can easily check that $\diamond_{0}$ is the Wick product and $\diamond_{1}$ is the Wiener product. Moreover, we note that

$$
\phi \cdot \psi=\mathcal{G}_{\frac{1}{2}, 1}\left(\mathcal{G}_{-\frac{1}{2}, 1} \phi \diamond \mathcal{G}_{-\frac{1}{2}, 1} \psi\right), \quad \phi, \psi \in(E) .
$$

A similar result can be found in $[7, \S 8.4]$. From this we obtain

$$
\phi \diamond_{\gamma} \psi=\mathcal{G}_{\frac{\gamma}{2}, 1}\left(\mathcal{G}_{-\frac{\gamma}{2}, 1} \phi \diamond \mathcal{G}_{-\frac{\gamma}{2}, 1} \psi\right), \quad \phi, \psi \in(E) .
$$

Lemma 3.2. For any $\phi \in(E)$ and $\xi, \eta \in E_{\mathbb{C}}$, we obtain

$$
\left\langle\left\langle\phi \diamond_{\gamma} \varphi_{\xi}, \varphi_{\eta}\right\rangle\right\rangle=e^{\langle\xi, \eta\rangle}\left\langle\left\langle\phi, \varphi_{\gamma \xi+\eta}\right\rangle\right\rangle .
$$


Proof. For $\xi, \eta, \zeta \in E_{\mathbb{C}}$, we observe that

$$
\left\langle\left\langle\varphi_{\zeta} \diamond_{\gamma} \varphi_{\xi}, \varphi_{\eta}\right\rangle\right\rangle=e^{\gamma\langle\zeta, \xi\rangle}\left\langle\left\langle\varphi_{\zeta+\xi}, \varphi_{\eta}\right\rangle\right\rangle=e^{\langle\xi, \eta\rangle}\left\langle\left\langle\varphi_{\zeta}, \varphi_{\gamma \xi+\eta}\right\rangle\right\rangle
$$

Since $\left\{\varphi_{\zeta} ; \zeta \in E_{\mathbb{C}}\right\}$ spans a dense subspace of $(E)$, the proof follows.

Proposition 3.3. There exists a unique separately continuous bilinear map $B$ : $(E)^{*} \times(E) \rightarrow(E)^{*}$ such that

(i) $B(\phi, \psi)=\phi \diamond_{\gamma} \psi, \quad \phi, \psi \in(E)$.

(ii) $\left\langle\left\langle B\left(\Phi, \varphi_{\xi}\right), \varphi_{\eta}\right\rangle\right\rangle=e^{\langle\xi, \eta\rangle}\left\langle\left\langle\Phi, \varphi_{\gamma \xi+\eta}\right\rangle\right\rangle, \quad \Phi \in(E)^{*}, \xi, \eta \in E_{\mathbb{C}}$.

Proof. For $\Phi \in(E)^{*}$ define a $\mathbb{C}$-valued function $F_{\Phi}$ by

$$
F_{\Phi}(\xi, \eta)=e^{\langle\xi, \eta\rangle}\left\langle\left\langle\Phi, \varphi_{\gamma \xi+\eta}\right\rangle\right\rangle, \quad \xi, \eta \in E_{\mathbb{C}} .
$$

Then $(z, w) \mapsto F_{\Phi}\left(z \xi+\xi^{\prime}, w \eta+\eta^{\prime}\right)$ is clearly an entire function on $\mathbb{C} \times \mathbb{C}$ for any $\xi, \xi^{\prime}, \eta, \eta^{\prime} \in E_{\mathbb{C}}$. Let $p \geq 0$ be such that $\|\Phi\|_{-p}<\infty$. Then we obtain

$$
\left|F_{\Phi}(\xi, \eta)\right| \leq\|\Phi\|_{-p} e^{a\left(|\xi|_{p}^{2}+|\eta|_{p}^{2}\right)}, \quad \xi, \eta \in E_{\mathbb{C}},
$$

where $a=\max \left\{\frac{1}{2} \rho^{2 p}+|\gamma|^{2}, \frac{1}{2} \rho^{2 p}+1\right\}$. Hence by Theorem 2.1, there exists a unique operator $\Xi_{\Phi} \in \mathcal{L}\left((E),(E)^{*}\right)$ such that

$$
\left\langle\left\langle\Xi_{\Phi} \varphi_{\xi}, \varphi_{\eta}\right\rangle\right\rangle=e^{\langle\xi, \eta\rangle}\left\langle\left\langle\Phi, \varphi_{\gamma \xi+\eta}\right\rangle\right\rangle, \quad \xi, \eta \in E_{\mathbb{C}} .
$$

Theorem 2.1 also says that for any $q>p$ with $2 a e^{2}\left\|A^{-2(q-p)}\right\|_{\mathrm{HS}}^{2}<1$, we have

$$
\left\|\Xi_{\Phi}(\phi)\right\|_{-q} \leq\left(1-2 a e^{2}\left\|A^{-2(q-p)}\right\|_{\mathrm{HS}}^{2}\right)^{-1}\|\Phi\|_{-p}\|\phi\|_{q}, \quad \phi \in(E) .
$$

Define a map $B:(E)^{*} \times(E) \rightarrow(E)^{*}$ by

$$
B(\Phi, \phi)=\Xi_{\Phi}(\phi), \quad \Phi \in(E)^{*}, \phi \in(E) .
$$

Then for each $\Phi \in(E)^{*}, B(\Phi, \cdot)=\Xi_{\Phi}$ is clearly a continuous linear operator on $(E)$. And it can be easily shown that for each $\phi \in(E), B(\cdot, \phi)$ is a linear operator on $(E)^{*}$. Moreover by $(3.4), B(\cdot, \phi)$ is continuous on $(E)^{*}$ for each $\phi \in(E)$. Hence the map $B$ is a separately continuous bilinear map of $(E)^{*} \times(E)$ into $(E)^{*}$.

Property (ii) follows from (3.3). By (ii) and Lemma 3.2, we have

$$
\left\langle\left\langle B\left(\phi, \varphi_{\xi}\right), \varphi_{\eta}\right\rangle\right\rangle=e^{\langle\xi, \eta\rangle}\left\langle\left\langle\phi, \varphi_{\gamma \xi+\eta}\right\rangle\right\rangle=\left\langle\left\langle\phi \diamond_{\gamma} \varphi_{\xi}, \varphi_{\eta}\right\rangle\right\rangle, \quad \phi \in(E), \xi, \eta \in E_{\mathbb{C}} .
$$

Hence $B\left(\phi, \varphi_{\xi}\right)=\phi \diamond_{\gamma} \varphi_{\xi}$ for $\xi \in E_{\mathbb{C}}$. This implies that $B$ satisfies (i).

The uniqueness of $B$ is clear from (i).

In Proposition 3.3, we write $B(\Phi, \phi)=\Phi \diamond_{\gamma} \phi=\phi \diamond_{\gamma} \Phi$ for any $\Phi \in(E)^{*}$ and $\phi \in(E)$. With this notation we have

$$
\left\langle\left\langle\Phi \diamond_{\gamma} \varphi_{\xi}, \varphi_{\eta}\right\rangle\right\rangle=e^{\langle\xi, \eta\rangle}\left\langle\left\langle\Phi, \varphi_{\gamma \xi+\eta}\right\rangle\right\rangle, \quad \xi, \eta \in E_{\mathbb{C}} .
$$

\section{FirST ORDER $\gamma$-DIFFERENTIAL OPERATORS}

We begin with the following lemma.

Lemma 4.1 ([1]). For $\Phi \in\left(E_{\mathbb{C}} \otimes(E)\right)^{*}$ and $\xi \in E_{\mathbb{C}}$, there exists a unique $\langle\Phi, \xi\rangle \in$ $(E)^{*}$ such that

$$
\langle\langle\langle\Phi, \xi\rangle, \phi\rangle\rangle=\langle\langle\Phi, \xi \otimes \phi\rangle\rangle, \quad \phi \in(E) .
$$

Further, $\langle\cdot, \cdot\rangle$ is a continuous bilinear map from $\left(E_{\mathbb{C}} \otimes(E)\right)^{*} \times E_{\mathbb{C}}$ into $(E)^{*}$. 
Proposition 4.2. For $\Xi \in \mathcal{L}\left((E),(E)^{*}\right)$ and $\Phi \in\left(E_{\mathbb{C}} \otimes(E)\right)^{*}$, the following statements are equivalent:

(i) $\Xi\left(\left\langle:{ }^{\otimes n}:, \xi^{\otimes n}\right\rangle\right)=n\left\langle: . \otimes(n-1):, \xi^{\otimes(n-1)}\right\rangle \diamond_{\gamma}\langle\Phi, \xi\rangle, \quad n \geq 0, \xi \in E_{\mathbb{C}}$.

(ii) $\Xi\left(\langle\cdot, \xi\rangle^{\diamond_{\gamma} n}\right)=n\langle\cdot, \xi\rangle^{\diamond_{\gamma}(n-1)} \diamond_{\gamma}\langle\Phi, \xi\rangle, \quad n \geq 0, \xi \in E_{\mathbb{C}}$.

(iii) $\Xi \varphi_{\xi}=\langle\Phi, \xi\rangle \diamond_{\gamma} \varphi_{\xi}, \quad \xi \in E_{\mathbb{C}}$.

(iv) $\widehat{\Xi}(\xi, \eta)=e^{\langle\xi, \eta\rangle}\left\langle\left\langle\Phi, \xi \otimes \varphi_{\gamma \xi+\eta}\right\rangle\right\rangle, \quad \xi, \eta \in E_{\mathbb{C}}$.

Proof. (i) $\Rightarrow$ (iii) $\quad$ For any $\xi \in E_{\mathbb{C}}$, we have

$$
\begin{aligned}
\Xi \varphi_{\xi} & =\Xi\left(\sum_{n=0}^{\infty} \frac{1}{n !}\left\langle:{ }^{\otimes n}:, \xi^{\otimes n}\right\rangle\right) \\
& =\sum_{n=1}^{\infty} \frac{1}{n !} n\left\langle: \cdot{ }^{\otimes(n-1)}:, \xi^{\otimes(n-1)}\right\rangle \diamond_{\gamma}\langle\Phi, \xi\rangle=\varphi_{\xi} \diamond_{\gamma}\langle\Phi, \xi\rangle,
\end{aligned}
$$

from which the assertion follows.

(ii) $\Rightarrow$ (iii) Since $\mathcal{G}_{\alpha, 1}\langle\cdot, \xi\rangle=\langle\cdot, \xi\rangle$ for any $\xi \in E_{\mathbb{C}}$ and $\alpha \in \mathbb{C}$, (3.2) implies that

$$
\langle\cdot, \xi\rangle^{\diamond}{ }^{\gamma}=\mathcal{G}_{\frac{\gamma}{2}, 1}\left(\left(\mathcal{G}_{-\frac{\gamma}{2}, 1}\langle\cdot, \xi\rangle\right)^{\diamond n}\right)=\mathcal{G}_{\frac{\gamma}{2}, 1}\left(\langle\cdot, \xi\rangle^{\diamond n}\right)=\mathcal{G}_{\frac{\gamma}{2}, 1}\left(\left\langle: .{ }^{\otimes n}:, \xi^{\otimes n}\right\rangle\right) .
$$

Thus for $\xi \in E_{\mathbb{C}}$ we obtain

$$
\sum_{n=0}^{\infty} \frac{1}{n !}\langle\cdot, \xi\rangle^{\diamond_{\gamma} n}=\mathcal{G}_{\frac{\gamma}{2}, 1}\left(\sum_{n=0}^{\infty} \frac{1}{n !}\left\langle: \cdot{ }^{\otimes n}:, \xi^{\otimes n}\right\rangle\right)=\mathcal{G}_{\frac{\gamma}{2}, 1} \varphi_{\xi}=e^{\frac{\gamma}{2}\langle\xi, \xi\rangle} \varphi_{\xi}
$$

By applying $\Xi$ to both sides of (4.6), we have

$$
\begin{aligned}
e^{\frac{\gamma}{2}\langle\xi, \xi\rangle} \Xi \varphi_{\xi} & =\Xi\left(\sum_{n=0}^{\infty} \frac{1}{n !}\langle\cdot, \xi\rangle^{\diamond_{\gamma} n}\right) \\
& =\sum_{n=1}^{\infty} \frac{1}{n !} n\langle\cdot, \xi\rangle^{\diamond_{\gamma}(n-1)} \diamond_{\gamma}\langle\Phi, \xi\rangle \\
& =\sum_{n=0}^{\infty} \frac{1}{n !}\langle\cdot, \xi\rangle^{\diamond_{\gamma} n} \diamond_{\gamma}\langle\Phi, \xi\rangle=e^{\frac{\gamma}{2}\langle\xi, \xi\rangle} \varphi_{\xi} \diamond_{\gamma}\langle\Phi, \xi\rangle .
\end{aligned}
$$

(iii) $\Rightarrow$ (i) Now fix $\xi \in E_{\mathbb{C}}$ and $\phi \in(E)$. Then for any $t \in \mathbb{C}$ we have

$$
\left\langle\left\langle\Xi \varphi_{t \xi}, \phi\right\rangle\right\rangle=\sum_{n=0}^{\infty} \frac{1}{n !}\left\langle\left\langle\Xi\left(\left\langle: .{ }^{\otimes n}:, \xi^{\otimes n}\right\rangle\right), \phi\right\rangle\right\rangle t^{n} .
$$

On the other hand, we have

$$
\left\langle\left\langle\langle\Phi, t \xi\rangle \diamond_{\gamma} \varphi_{t \xi}, \phi\right\rangle\right\rangle=\sum_{n=0}^{\infty} \frac{1}{n !}\left\langle\left\langle\left\langle:{ }^{\otimes n}:, \xi^{\otimes n}\right\rangle \diamond_{\gamma}\langle\Phi, \xi\rangle, \phi\right\rangle t^{n+1} .\right.
$$

By comparing (4.7) with (4.8), we prove the assertion.

(iii) $\Rightarrow$ (ii) $\operatorname{Fix} \xi \in E_{\mathbb{C}}$ and $\phi \in(E)$ again. Then we have

$$
e^{\frac{\gamma}{2} t^{2}\langle\xi, \xi\rangle}\left\langle\left\langle\Xi \varphi_{t \xi}, \phi\right\rangle\right\rangle=\sum_{n=0}^{\infty} \frac{1}{n !}\left\langle\left\langle\Xi\left(\langle\cdot, \xi\rangle^{\diamond_{\gamma} n}\right), \phi\right\rangle\right\rangle t^{n},
$$


and

$$
\begin{aligned}
e^{\frac{\gamma}{2} t^{2}\langle\xi, \xi\rangle}\left\langle\left\langle\langle\Phi, t \xi\rangle \diamond_{\gamma} \varphi_{t \xi}, \phi\right\rangle\right\rangle & =\left\langle\left\langle\left\langle\Phi, t \xi \diamond_{\gamma} \sum_{n=0}^{\infty} \frac{1}{n !}\langle\cdot, t \xi\rangle^{\diamond_{\gamma} n}, \phi\right\rangle\right\rangle\right. \\
& =\sum_{n=0}^{\infty} \frac{1}{n !}\left\langle\left\langle\langle\cdot, \xi\rangle^{\diamond_{\gamma} n} \diamond_{\gamma}\langle\Phi, \xi\rangle, \phi\right\rangle\right\rangle t^{n+1} .
\end{aligned}
$$

By comparing the above two equations, the assertion is proved.

(iii) $\Leftrightarrow$ (iv) is clear from the fact that

$$
\left\langle\left\langle\langle\Phi, \xi\rangle \diamond_{\gamma} \varphi_{\xi}, \varphi_{\eta}\right\rangle\right\rangle=e^{\langle\xi, \eta\rangle}\left\langle\left\langle\Phi, \xi \otimes \varphi_{\gamma \xi+\eta}\right\rangle\right\rangle, \quad \xi, \eta \in E_{\mathbb{C}} .
$$

Theorem 4.3. For $\Phi \in\left(E_{\mathbb{C}} \otimes(E)\right)^{*}$, there is a unique operator $\Xi \in \mathcal{L}\left((E),(E)^{*}\right)$ such that one of conditions (i)-(iv) in Proposition 4.2 holds.

Proof. This follows immediately from the fact that the function

$$
F(\xi, \eta)=e^{\langle\xi, \eta\rangle}\left\langle\left\langle\Phi, \xi \otimes \varphi_{\gamma \xi+\eta}\right\rangle\right\rangle, \quad \xi, \eta \in E_{\mathbb{C}},
$$

satisfies (S1) and (S2) in Theorem 2.1.

Definition 4.4. The operator $\Xi$ given as in Theorem 4.3 is called a first order $\gamma$-differential operator with coefficient $\Phi \in\left(E_{\mathbb{C}} \otimes(E)\right)^{*}$.

This operator $\Xi$ has the following integral expression:

$$
\Xi=\int_{T} \Phi(t) \diamond_{\gamma} \partial_{t} d t
$$

The proof of the next theorem can be done by a simple modification of the proof of Theorem 3.4 given in [1].

Theorem 4.5. Let $\Xi \in \mathcal{L}\left((E),(E)^{*}\right)$ be a first order $\gamma$-differential operator with coefficient $\Phi \in\left(E_{\mathbb{C}} \otimes(E)\right)^{*}$. Then $\Xi \in \mathcal{L}((E),(E))$ if and only if $\Phi \in E_{\mathbb{C}}^{*} \otimes(E)$.

Example 4.6. (1) For any $y \in E_{\mathbb{C}}, D_{y} \in \mathcal{L}((E),(E))$ is a first order $\gamma$-differential operator with coefficient $y \otimes 1 \in E_{\mathbb{C}}^{*} \otimes(E)$.

(2) Let $\Phi_{0} \in\left(E_{\mathbb{C}} \otimes(E)\right)^{*}$ be given by the formal formula

$$
\Phi_{0}(t, x)=x(t), \quad t \in T, x \in E^{*} .
$$

That is, $\left\langle\left\langle\Phi_{0}, \xi \otimes \phi\right\rangle\right\rangle=\left\langle\xi, f_{1}\right\rangle$ for any $\xi \in E_{\mathbb{C}}$ and $\phi \sim\left(f_{n}\right) \in(E)$. Then $\gamma \Delta_{G}+N$ is a first order $\gamma$-differential operator with coefficient $\Phi_{0}$.

Definition 4.7. Let $\Xi \in \mathcal{L}\left((E),(E)^{*}\right)$. Then $\Xi$ is said to be a $\gamma$-derivation if the following holds:

$$
\Xi\left(\phi \diamond_{\gamma} \psi\right)=(\Xi \phi) \diamond_{\gamma} \psi+\phi \diamond_{\gamma}(\Xi \psi), \quad \phi, \psi \in(E) .
$$

By using the fact that the set of exponential vectors spans a dense subspace of $(E)$, we obtain the following proposition:

Proposition 4.8. Let $\Xi \in \mathcal{L}\left((E),(E)^{*}\right)$. Then $\Xi$ is a $\gamma$-derivation if and only if for any $\xi, \eta, \zeta \in E_{\mathbb{C}}$, we have

$$
e^{\gamma\langle\xi, \eta\rangle} \widehat{\Xi}(\xi+\eta, \zeta)=e^{\langle\eta, \zeta\rangle} \widehat{\Xi}(\xi, \gamma \eta+\zeta)+e^{\langle\xi, \zeta\rangle} \widehat{\Xi}(\eta, \gamma \xi+\zeta) .
$$

Theorem 4.9. Let $\Xi \in \mathcal{L}\left((E),(E)^{*}\right)$. Then $\Xi$ is a $\gamma$-derivation if and only if $\Xi$ is a first order $\gamma$-differential operator with some coefficient $\Phi \in\left(E_{\mathbb{C}} \otimes(E)\right)^{*}$. 
Proof. Let $\Xi \in \mathcal{L}\left((E),(E)^{*}\right)$ be a first order $\gamma$-differential operator with coefficient $\Phi \in\left(E_{\mathbb{C}} \otimes(E)\right)^{*}$. Then it can be shown that $\widehat{\Xi}(\xi, \eta)=e^{\langle\xi, \eta\rangle}\left\langle\left\langle\Phi, \xi \otimes \varphi_{\gamma \xi+\eta}\right\rangle\right\rangle$ satisfies (4.9), and hence $\Xi$ is a $\gamma$-derivation.

Now assume that $\Xi \in \mathcal{L}\left((E),(E)^{*}\right)$ is a $\gamma$-derivation. Since $\xi \mapsto\langle\cdot, \xi\rangle$ is continuous and linear, we see that $\xi \mapsto \Xi(\langle\cdot, \xi\rangle)$ is a continuous linear map from $E_{\mathbb{C}}$ into $(E)^{*}$. Hence there exists a unique $\Phi \in\left(E_{\mathbb{C}} \otimes(E)\right)^{*}$ such that

$$
\langle\Phi, \xi\rangle=\Xi(\langle\cdot, \xi\rangle), \quad \xi \in E_{\mathbb{C}} .
$$

Since $\Xi$ is a $\gamma$-derivation, we have for any $n \geq 0$

$$
\Xi\left(\langle\cdot, \xi\rangle^{\diamond_{\gamma} n}\right)=n\langle\cdot, \xi\rangle^{\diamond_{\gamma}(n-1)} \diamond_{\gamma} \Xi(\langle\cdot, \xi\rangle)=n\langle\cdot, \xi\rangle^{\diamond_{\gamma}(n-1)} \diamond_{\gamma}\langle\Phi, \xi\rangle, \quad \xi \in E_{\mathbb{C}} .
$$

Hence by Proposition 4.2 and Theorem $4.3, \Xi$ is a first order $\gamma$-differential operator with coefficient $\Phi \in\left(E_{\mathbb{C}} \otimes(E)\right)^{*}$.

\section{Characterization of the operator $\gamma \Delta_{G}+N$}

We shall adopt the notion of rotation-invariance of operators given in $[9, \S 5.4]$. Let $O(E ; H)$ is the space of rotations on $E$, that is, $O(E ; H)$ is the set of linear homeomorphism from $E$ onto itself such that $|g \xi|_{0}=|\xi|_{0}, \xi \in E$.

Recall from $[9, \S 5.4]$ that $\Xi \in \mathcal{L}\left((E),(E)^{*}\right)$ is rotation-invariant if and only if

$$
\widehat{\Xi}(\xi, \eta)=\widehat{\Xi}(g \xi, g \eta), \quad \xi, \eta \in E_{\mathbb{C}}, g \in O(E ; H),
$$

and that $F \in\left(E_{\mathbb{C}}^{\otimes n}\right)^{*}$ is rotation-invariant if and only if

$$
\left\langle F, \xi_{1} \otimes \cdots \otimes \xi_{n}\right\rangle=\left\langle F, g \xi_{1} \otimes \cdots \otimes g \xi_{n}\right\rangle, \quad \xi_{1}, \cdots, \xi_{n} \in E_{\mathbb{C}}, g \in O(E ; H) .
$$

We note that $\Delta_{G}$ and $N$ are rotation-invariant.

Theorem 5.1. Let $\Xi \in \mathcal{L}((E),(E))$. Then the following are equivalent:

(i) $\Xi$ is a constant multiple of $\gamma \Delta_{G}+N$.

(ii) $\Xi$ is a first order $\gamma$-differential operator with coefficient $k \Phi_{0}$ for some $k \in \mathbb{C}$, where $\Phi_{0}$ is given as in Example 4.6, (2).

(iii) $\Xi$ is a rotation-invariant $\gamma$-derivation.

In order to prove Theorem 5.1, we need the following lemma, which can be proved by Theorem 5.5.4 in [9].

Lemma 5.2. Let $F$ be a non-zero rotation-invariant element in $E_{\mathbb{C}}^{*} \otimes E_{\mathbb{C}}^{\widehat{\otimes} n}$. Then $n$ must be 1 , and $F=k \tau$ for some constant $k \in \mathbb{C}$. Here $\tau \in E_{\mathbb{C}}^{*} \otimes E_{\mathbb{C}}$ is given by $\langle\tau, \xi \otimes \eta\rangle=\langle\xi, \eta\rangle, \xi, \eta \in E_{\mathbb{C}}$.

Proof of Theorem 5.1. In view of Example 4.6 (2) and Definition 4.4, (i) is equivalent to (ii). The proof of (i) $\Rightarrow$ (iii) is clear from Proposition 4.8. To complete the proof, we need to prove that (iii) $\Rightarrow$ (ii).

Let $\Xi \in \mathcal{L}((E),(E))$ be a rotation-invariant $\gamma$-derivation. Then by Theorems 4.9 and 4.5 , there exists a $\Phi \in E_{\mathbb{C}}^{*} \otimes(E)$ such that $\Xi$ is a first order $\gamma$-differential operator with coefficient $\Phi$. The rotation-invariance of $\Xi$ implies

$$
\left\langle\left\langle\Phi, \xi \otimes \varphi_{\gamma \xi+\eta}\right\rangle\right\rangle=\left\langle\left\langle\Phi, g \xi \otimes \varphi_{g(\gamma \xi+\eta)}\right\rangle\right\rangle, \quad \xi, \eta \in E_{\mathbb{C}}, g \in O(E ; H),
$$

or equivalently

$$
\left\langle\left\langle\Phi, \xi \otimes \varphi_{\eta}\right\rangle\right\rangle=\left\langle\left\langle\Phi, g \xi \otimes \varphi_{g \eta}\right\rangle\right\rangle, \quad \xi, \eta \in E_{\mathbb{C}}, g \in O(E ; H) .
$$


In view of Proposition 6.3.1 in [9], there exists a unique sequence $\left\{F_{n}\right\}, F_{n} \in E_{\mathbb{C}}^{*} \otimes$ $E_{\mathbb{C}}^{\widehat{\otimes} n}$, such that

$$
\left\langle\left\langle\Phi, \xi \otimes \varphi_{\eta}\right\rangle\right\rangle=\sum_{n=0}^{\infty}\left\langle F_{n}, \xi \otimes \eta^{\otimes n}\right\rangle, \quad \xi, \eta \in E_{\mathbb{C}} .
$$

From this we have, for any $\xi, \eta \in E_{\mathbb{C}}$ and $g \in O(E ; H)$,

$$
\sum_{n=0}^{\infty}\left\langle F_{n}, \xi \otimes \eta^{\otimes n}\right\rangle t^{n}=\sum_{n=0}^{\infty}\left\langle F_{n}, g^{\otimes(n+1)}\left(\xi \otimes \eta^{\otimes n}\right)\right\rangle t^{n}, \quad t \in \mathbb{C} .
$$

Hence for any $n \geq 0$ we have

$$
\left\langle F_{n}, \xi \otimes \eta^{\otimes n}\right\rangle=\left\langle F_{n}, g^{\otimes(n+1)}\left(\xi \otimes \eta^{\otimes n}\right)\right\rangle, \quad \xi, \eta \in E_{\mathbb{C}}, g \in O(E ; H) .
$$

Since $F_{n} \in E_{\mathbb{C}}^{*} \otimes E_{\mathbb{C}}^{\widehat{\otimes} n}, F_{n}$ is rotation-invariant. But by Lemma $5.2, F_{n}=0$ for $n \neq 1$ and $F_{1}=k \tau$ for some constant $k \in \mathbb{C}$. Hence in view of Example 4.6 (2) and (5.10), we have $\Phi=k \Phi_{0}$.

Remark. (1) It is noteworthy that $\frac{1}{\gamma}\left(\gamma \Delta_{G}+N\right)$ converges to $\Delta_{G}$ (a second order differential operator) in $\mathcal{L}((E),(E))$ as $\gamma \rightarrow \infty$ (see [2]).

(2) For each $\gamma \in \mathbb{C}$, the operator $\gamma \Delta_{G}+N$ corresponds a one-parameter transformation group on $E^{*}$ (see [3]).

(3) Theorem 5.1 for the cases $\gamma=1$ and $\gamma=0$ is found in the recent paper [4].

\section{REFERENCES}

[1] D. M. Chung and T. S. Chung, Wick derivations on white noise functionals, J. Korean Math. Soc. 33(1996), No. 4, 993-1008. CMP 97:05

[2] D. M. Chung, T. S. Chung and U. C. Ji, A characterization theorem for operators on white noise functionals, preprint.

[3] D. M. Chung and U. C. Ji, Transformation groups on white noise functionals and their applications, to appear in J. Appl. Math. Optim.

[4] S. W. He, J. G. Wang and R. Q. Yao, The characterizations of Laplacians in white noise analysis, Nagoya Math. J. 143(1996), 93-109. CMP 97:02

[5] T. Hida, H.-H. Kuo and N. Obata, Transformations for white noise functionals, J. Funct. Anal. 111(1993), 259-277. MR 93m:46042

[6] T. Hida, H.-H. Kuo, J. Potthoff and L. Streit, "White Noise: An Infinite Dimensional Calculus," Kluwer Academic, 1993. MR 95f:60046

[7] H.-H. Kuo, "White Noise Distribution Theory," CRC Press, 1996. CMP 96:12

[8] N. Obata, Rotation-invariant operators on white noise functionals, Math. Z. 210(1992), 6989. MR 93m: 46048

[9] N. Obata, "White noise calculus and Fock space," Lect. Notes in Math. Vol. 1577, SpringerVerlag, 1994. MR 96e:60061

[10] N. Obata, Derivations on white noise functionals, Nagoya Math. J. 139(1995), 21-36. MR 97m: 46073

[11] J. Potthoff and L. Streit, A characterization of Hida distributions, J. Funct. Anal. 101(1991), 212-229. MR 93a:46078

Department of Mathematics, Sogang University, Seoul, 121-742, Korea

E-mail address: dmchung@ccs.sogang.ac.kr 